\title{
Simulation of localization failure with strain-gradient-enhanced damage mechanics
}

\author{
Weiyuan Zhou*, ${ }^{*}$, Jidong Zhao ${ }^{1}$, Yuangao Liu and Qiang Yang \\ Department of Hydraulic Engineering, Tsinghua University, Beijing 100084, People's Republic of China
}

\begin{abstract}
SUMMARY
Strain gradient implies an important characteristic in localized damage deformation, which can be observed in the softening state of brittle materials, and strain gradients constitute the basic behaviours of localization failure area of the materials. The most important point in strain gradient is its damaging function including an internal length scale, which can be used to express the scale effects of mechanical responses of brittle rock mass. By extending the strain gradient theory and introducing an intrinsic material length scale into the constitutive law, the authors develop an isotropic damage model as well as a microcrack-based anisotropic damage model for rock-like materials in this paper. The proposed models were used to simulate the damage localization under uniaxial tension and plain strain compression, respectively. The simulated results well illustrated the potential of these models in dealing with the well-known meshsensitivity problem in FEM. In the computation, elements with $C_{1}$ continuity have been implemented to incorporate the proposed models for failure localization. When regular rectangle elements are encountered, the coupling between finite difference method (FDM) and conventional finite element method (FEM) is used to avoid large modification to the existing FEM code, and to obtain relatively higher efficiency and reasonably good accuracy. Application of the anisotropic model to the 3D-non-linear FEM analysis of Ertan arch dam has been conducted and the results of its numerical simulation coincide well with those from the failure behaviours obtained by Ertan geophysical model test. In this paper, new applications of gradient theories and models for a feasible approach to simulate localized damage in brittle materials are presented. Copyright (C) 2002 John Wiley \& Sons, Ltd.
\end{abstract}

KEY WORDS: strain gradient theory; damage localization; finite element method (FEM); finite difference method (FDM); mesh dependence

\section{INTRODUCTION}

Localized failure of geomaterials is a very common phenomenon and has attracted much interest from scholars. Rudnicki and Rice [1], Ottosen and Runesson [2] and Benallal [3] treated the localized shear band as a phenomenon of bifurcation. For rate-independent material,

\footnotetext{
*Correspondence to: Weiyuan Zhou, Department of Hydraulic Engineering, Tsinghua University, Beijing 100084, People's Republic of China.

†E-mail: zhouwy@tsinghua.edu.cn

${ }^{1}$ E-mail: zhaojd97@mails.tsinghua.edu.cn
}

Contract/grant sponsor: National Science Foundation of China (NSFC); contract/grant number: 59939190. Contract/ grant sponsor: Schlumberger BGC

Copyright (C) 2002 John Wiley \& Sons, Ltd.

Received 12 September 2001

Revised 19 March 2002 
localized shear band is closely related to the unsteady behaviour of material. The incipience of localized failure corresponds to the losts of ellipticity of the governing equation as well as the uniqueness of the solution, and simultaneously the strain field becomes discontinuous, with large flow rate and strain gradient in the band-like region. So far how to simulate and predict the failure process - pre- and post-failure of brittle rock-like materials is still a focus. It consists of several important points such as: formation of shear bands in rock-like materials at softening state; fracture initiation, nucleation and propagation with intense strain gradients; the mechanics meaning of gradient in deformations of rock materials under external loadings; the physical meaning and mechanical role of the intrinsic material length and its relation with localization formation.

On dealing with the problem of localization, the conventional continuum mechanics is always used by scholars. However, this method comes out to be inadequate to identify the characterized behaviours of localization. In fact, during the localized failure state of rock-like materials, the strain gradient makes a crucial proportion of failure-driven force and becomes the controlling factor of both localization condition and formation. Whereas, the conventional continuum mechanics neglects the contribution of strain gradient to localization and has no intrinsic material length in the constitutive law, which leads to such problems as zero-energy dissipation and mesh dependence in the FEM simulation of localization, the incapability of determining the direction and size of localized band and the indetermination of computation results in the failure state.

To account for the influence of gradient items and avoid the deficiency of conventional continuum mechanics on the problem of localization, different gradient models have been developed. Generally, these gradient models can be classified into three types. The first kind directly considers the gradient effects of damage variables within the continuum damage mechanics, which has a similar purpose of the gradient of strain. E.g. Comi [4] developed an isotropic gradient-enhanced damage model, in which the loading function not only depends on the damage value, but also on its Laplacian. Fremond and Nedjar [5] proposed a theory of continuum damage mechanics within the framework of the principle of virtual work, taking into account the influence of damage velocity and its gradient on the work of the internal forces. However, the physical meaning of damage gradients still cannot be clearly interpreted.

The second kind gradient model belongs to the non-local gradient theory. This kind of gradient model is pioneered by Muhlhaus and Vardoulakis [6], de Borst and Muhlhaus [7] and Bazant et al. [8-11]. They proposed a micro-plane-theory-based non-local model to analyse the size effect of structure fracture. Peelings et al. [12-14] and Geers et al. [15,16] further developed a gradient damage model by connecting local with non-local equivalent strains. Kuhl and Ramm [17] made a combination of the above two models and proposed an anisotropic gradientenhanced damage model. Tvergaard and Needleman [18] adopted non-local method with intrinsic material length scale included to minimize the mesh-sensitive problem for ductile porous media. The gradient term of non-local model is derived from the spatial average of strain, and at the same time, a material length scale is introduced. This model is successful in predicting the localization formation in rock-like materials. However, it has its weak points since this method leads to the weakening of total stiffness of materials due to the spatial average of strain, which lowers the critical hardening modulus for localized bifurcation and makes the computation results safer than the actual behaviour. As a result, its numerical simulation cannot reflect the true failure process and the actual limit. 
The third kind can be called the local gradient model. Papers representing this kind of model are presented by Cosserats [19], Toupin [20], Mindlin [21,22], Aifantis [23,24], Muhlhaus and Aifantis [25], Fleck and Hutchinson [26,27], Fleck et al. [28], Gao et al. [29], and so on. Local gradient model generally includes a rotation gradient or entire strain gradient in the model to construct the constitutive law. An intrinsic material length is also introduced into the law to balance the equations. Local gradient model experiences a process from elastic gradient models to elasto-plastic models. These models mainly treat such materials as metals. In fact, for rocklike geomaterials, the characteristic of strain gradient during localization is rather evident (see References [30,31]). However, few literatures of local strain gradient model ever treat the problem of localization in these materials.

The motivation of this paper is to introduce the effect of local strain gradient into continuum damage mechanics and to account for those characteristic behaviours of localization that conventional continuum mechanics has difficulties to deal with. Continuum damage mechanics is an effective tool in describing the characteristic of anisotropy, shear dilatation and load-path dependence of geomaterials. Here the authors choose the local strain gradient form presented in the paper of Fleck and Hutchinson [26,27], and adopt a scalar variable and a second-order tensor, respectively, for the isotropic and the anisotropic damage models. In the following paragraphs the local strain gradient model of Fleck and Hutchinson [26,27] are briefly described. The combinations of the local strain gradient with the continuum damage mechanics are separately presented for the isotropic and anisotropic cases. These models were implemented in FEM and were used to simulate localized shear bands. The engineering application to Ertan arch dam by 3D-non-linear FEM analysis with the proposed models was also conducted with the anisotropic model. The computed failure results by this model were compared with the rupture behaviours from the geophysical model test of the dam.

As for notations and symbols, bold-face letters denote matrices and vectors; the symbol ':' denotes an inner product of two second-order tensors (e.g. $\mathbf{a}: \mathbf{b}=a_{i j} b_{i j}$ ), or a double contraction of adjacent indices of tensors of rank two and higher (e.g. $D: \varepsilon=D_{i j k l} \varepsilon_{k l}$ ); the symbol ' $:$ ' denotes an inner product of two third-order tensors (e.g. c $\vdots \mathbf{d}=c_{i j k} d_{i j k}$ ), or a triple contraction of adjacent indices of tensors of rank three and higher (e.g. $\boldsymbol{\Lambda} \vdots \boldsymbol{\eta}=\Lambda_{i j k l m n} \eta_{l m n}$ ); the rate of change with respect to time is denoted by an ascent dot (e.g. $\dot{d}$ ).

\section{GRADIENT-ENHANCED DAMAGE MODEL}

\subsection{Isotropic damage model}

2.1.1. Conventional continuum model: mesh dependence. The conventional continuum theories generally neglect the effects of strain gradient in the process analysis of localized failure. Although applicable for most situations, they are no longer sufficient to describe the localized failure of strain-softening materials, like rock and concrete. FEM simulation based on these theories will pose an obvious mesh-dependence problem, which can be seen from the following numerical samples (Figure 1). In the example, three different meshes were used to study the damage and failure behaviour of bars under uniaxial tension. An isotropic damage constitutive law proposed by Simo and Ju [32] is applied to model them. The numbers of the mesh are 33, 90 and 550, respectively, and all the other conditions are the same. To trigger the localization, the magnitudes of some material parameters are slightly decreased in the middle elements. As 


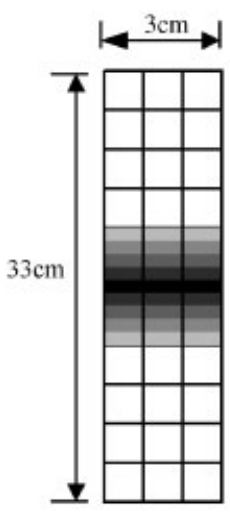

(a) : $3 \times 11$

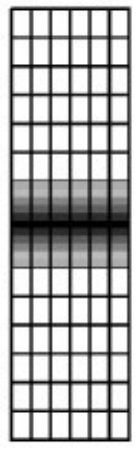

(b) : $6 \times 15$

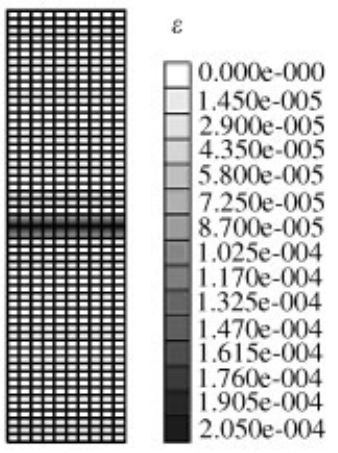

(c) : $10 \times 55$

Figure 1. FEM simulations of samples under uniaxial tension: based on the conventional damage model, the strain distribution has a strong dependence on the mesh discretization.

shown in Figure 1, a strong mesh dependence of strain distribution is obtained by FEM simulation. This phenomenon is incorrigible under the framework of conventional constitutive law due to the neglect of strain gradient and the lack of intrinsic length scale of material in the constitutive law.

2.1.2. Isotropic gradient-enhanced damage model. The strain gradient theory proposed by Fleck and Hutchinson [26,27] relates the strain and strain gradient to the displacement field $\mathbf{u}$ by

$$
\varepsilon_{i j}=\left(u_{i, j}+u_{j, i}\right) / 2, \quad \eta_{i j k}=\left(u_{k, i j}+u_{k, j i}\right) / 2
$$

Here, $\varepsilon_{i j}$ is the symmetric strain tensor, and $\eta_{i j k}$ is the symmetric third-order strain gradient tensor. The Cauchy stress $\sigma_{i j}$ and some higher-order stress $\tau_{i j k}$ are assumed to be the work conjugate of strain $\varepsilon_{i j}$ and strain gradient $\eta_{i j k}$, respectively. The higher-order stress should satisfy the following symmetric condition: $\tau_{i j k}=\tau_{j i k}$.

The strain gradient theory is originally developed within the framework of micro-mechanics and meso-mechanics. By extending it to the materials with damaged micro-structure and considering its macroscopic damage effect, the authors proposed an isotropic straingradient-enhanced damage model. On supposing that the isotropic damage variable of the material can be represented by a scalar $d$, the Helmholtz free energy function has the following form:

$$
\psi=\frac{1}{2}(1-d) \boldsymbol{\varepsilon}: \mathbf{D}^{\mathrm{e}}: \boldsymbol{\varepsilon}+\frac{1}{2}(1-d) \boldsymbol{\eta} \vdots \boldsymbol{\Lambda}^{\mathrm{e}}: \boldsymbol{\eta}+\int_{0}^{\kappa} \phi(\kappa) \mathrm{d} \widehat{\kappa}
$$

where: $\mathbf{D}^{\mathrm{e}}$ is the fourth-order elasticity tensor; $\boldsymbol{\Lambda}^{\mathrm{e}}$ is the sixth-order strain-gradient-related elasticity tensor, $\boldsymbol{\Lambda}_{i j k l m n}^{\mathrm{e}}=l^{2} D_{i j l m}^{\mathrm{e}} \delta_{k n}, \delta_{k n}$ is the Kronecker delta (see Reference [33]), and $\kappa$ is an additional internal variable. The third term $\int_{0}^{\kappa} \phi(\kappa) \mathrm{d} \widehat{\kappa}$ accounts for the softening/ hardening behaviour in the material. $l$ is an intrinsic material length with a dimension of length. Its magnitude is closely related to the micro-structure of the material. Generally, 
$l$ remains constant for the observed objects or test samples. Its value is considered to be only related to the initial micro-defects and can be obtained from the experiments. Massive amount of experiments had been done for ductile materials, especially for metals [28,34-41]. The material lengths obtained by experiments are: for copper, $l \approx 4 \mu \mathrm{m}$ and for nickel, $l \approx 6 \mu \mathrm{m}$. For rock-concrete-like materials, inhomogeneous micro-structure mainly refers to micro-cracks, micro-voids and granule of the material. Pan [30] obtained the material length for coal rock and sandstone by rock-burst test as $l \approx 0.53 \mathrm{~mm}$ for coal rock and $l \approx$ $0.47 \mathrm{~mm}$ for sandstone. Also, the length value of other harder rock is about 4-6 times larger than that of sandstone. For granite treated in this paper, the material length is adopted as $l \approx 1.5 \mathrm{~mm}$.

According to the Clausius-Duhem inequation, the Cauchy stress tensor $\sigma_{i j}$ and the higher-order stress tensor $\tau_{i j k}$ can be determined by the work conjugate of $\varepsilon_{i j}$ and $\eta_{i j k}$, respectively.

$$
\sigma_{i j}=\frac{\partial \psi}{\partial \varepsilon_{i j}}=(1-d) D_{i j k l}^{\mathrm{e}} \varepsilon_{k l}, \quad \tau_{i j k}=\frac{\partial \psi}{\partial \eta_{i j k}}=(1-d) l^{2} D_{i j k l}^{\mathrm{e}} \delta_{m n} \eta_{l m n}
$$

The above-mentioned Cauchy stress and higher-order stress satisfy the equilibrium equation in the absence of body force given below

$$
\nabla \cdot \boldsymbol{\sigma}-\nabla \cdot(\nabla \cdot \tau)=\mathbf{0}
$$

2.1.3. Damage evolution law. From Equation (2), the thermodynamic force $Y$ conjugate to damage variable $d$ can be derived as

$$
Y=-\frac{\partial \psi}{\partial d}=\frac{1}{2} \boldsymbol{\varepsilon}: \mathbf{D}^{\mathrm{e}}: \boldsymbol{\varepsilon}+\frac{1}{2} \boldsymbol{\eta} \vdots \boldsymbol{\Lambda}^{\mathrm{e}}: \boldsymbol{\eta}
$$

Note that (:) denotes the third-order tensor product operator.

Define a damage surface like the plasticity-yielding surface in the stress space as below [30]:

$$
g\left[Y, d, r_{t}\right]=G(Y)-r_{t}(d, \kappa) \leqslant 0
$$

where $G(Y)$ denotes the equivalent fracturing force and $r_{t}(d, \kappa)$ the representative radius characterizing the damage surface, which implies the dependence of fracturing and yielding behaviour on the accumulated damage magnitude in the material.

The flow rule of damage in the material analogous to that in plasticity theory is defined as

$$
\dot{d}=\dot{\gamma} \frac{\partial g}{\partial Y}
$$

The damage surface yields the Kuhn-Tucker conditions:

$$
g \leqslant 0, \quad \dot{\gamma} \geqslant 0, \quad g \dot{\gamma}=0
$$

and the consistency condition

$$
\dot{g} \dot{\gamma}=0
$$

Equations (6)-(9) imply that, within the damage surface, $\dot{d}=0$ always holds and no damage dissipation will be introduced for any loading/unloading process in the stress space. 
Generally, the rate of internal variable, $\dot{\kappa}$, can be implicitly assumed as $\dot{\kappa}=\dot{\gamma}$. Therefore, given the consistency condition of damage, one can have

$$
\begin{aligned}
\dot{g} & =\frac{\partial g}{\partial Y} \dot{Y}+\frac{\partial g}{\partial d} \dot{d}+\frac{\partial g}{\partial \kappa} \dot{\kappa} \\
& =\frac{\partial g}{\partial Y} \dot{Y}+\dot{\gamma} \frac{\partial g}{\partial d} \frac{\partial g}{\partial Y}+\dot{\gamma} \frac{\partial g}{\partial r_{t}} \frac{\partial r_{t}}{\partial \kappa} \\
& =\frac{\partial g}{\partial Y} \dot{Y}+\dot{\gamma}\left(\frac{\partial g}{\partial d} \frac{\partial g}{\partial Y}+\frac{\partial g}{\partial r_{t}} \frac{\partial r_{t}}{\partial \kappa}\right) \\
& =0
\end{aligned}
$$

Thus

$$
\dot{\gamma}=-{ }_{\varsigma} \dot{Y}\left(\frac{\partial g}{\partial d} \frac{\partial g}{\partial Y}+\frac{\partial g}{\partial r_{t}} \frac{\partial r_{t}}{\partial \kappa}\right)^{-1} \frac{\partial g}{\partial Y}
$$

where

$$
\varsigma= \begin{cases}1 & \text { when } g=0, \quad \frac{\partial g}{\partial Y} \dot{Y} \geqslant 0 \\ 0 & \text { else. }\end{cases}
$$

Hence, the general form of damage evolution law can be expressed as

$$
\dot{d}=-\varsigma \dot{Y} \frac{\partial g}{\partial Y}\left(\frac{\partial g}{\partial d} \frac{\partial g}{\partial Y}+\frac{\partial g}{\partial r_{t}} \frac{\partial r_{t}}{\partial \kappa}\right)^{-1} \frac{\partial g}{\partial Y}
$$

However, the above implicit form of damage evolution law is inconvenient for numerical implementation. An alternative simple explicit formulation is proposed in this paper. By the definition of damage, the thermodynamic force $\mathbf{Y}$ conjugated to the damage variable can be regarded as the energy-releasing rate in fracture mechanics. Consequently, the internal variable $\kappa$ can be determined explicitly in terms of the maximum value between a threshold value $\kappa_{0}$ and the energy release rate during the loading/unloading process in the following form [17]:

$$
\kappa=\max _{-\infty<t<\tau}\left(\left[\frac{1}{2} \mathbf{\varepsilon}(t): \mathbf{D}^{\mathrm{e}}: \mathbf{\varepsilon}(t)+\frac{1}{2} \boldsymbol{\eta}(t) \vdots \boldsymbol{\Lambda}^{\mathrm{e}} \vdots \mathbf{\eta}(t)\right], \kappa^{0}\right)
$$

A power distribution model proposed by Geers et al. $[15,16]$ is then used to determine the damage variable to characterize the strain-softening behaviour in rock-like material

$$
d=1-\left(\frac{\kappa_{0}}{\kappa}\right)^{\beta}\left(\frac{\kappa_{c}-\kappa}{\kappa_{c}-\kappa_{0}}\right)^{\alpha}
$$

where $\kappa_{c}$ is an internal hardening/softening variable representing the upper limit value of $\kappa$ when damage reaches maximum. The two exponents, $\alpha$ and $\beta$, reflect the slope and the shape of stressstrain softening curve while the damage threshold $\kappa_{0}$ mainly relates to the peak of the loading 
displacement curve. For convenience of computation, Equation (14) is further simplified by supposing $\beta=1$,

$$
\begin{aligned}
d=1-\frac{\kappa_{0}}{\kappa}\left(\frac{\kappa_{c}-\kappa}{\kappa_{c}-\kappa_{0}}\right)^{\alpha} & =1-\frac{\kappa_{0}}{\kappa}\left(1-\frac{\kappa-\kappa_{0}}{\kappa_{c}-\kappa_{0}}\right)^{\alpha} \\
& =1-\frac{\kappa_{0}}{\kappa}\left(1-\zeta\left(\kappa-\kappa_{0}\right)\right)^{\alpha}
\end{aligned}
$$

where $\zeta=1 /\left(\kappa_{c}-\kappa_{0}\right)$. An alternative form of damage evolution law proposed by Carmeliet and de Borst [42], which is more convenient to use, is adopted in the paper:

$$
d=\phi(\kappa)=1-\frac{\kappa_{0}}{\kappa}\left[1-\alpha+\alpha \mathrm{e}^{-\zeta\left(\kappa-\kappa_{0}\right)}\right]
$$

Figure 2 shows a relation curve for damage value, $d$, with the influencing parameters in (16). Figure 3 gives a sketch of the effect of $\alpha$ on the uniaxial stress and strain curve.

\subsection{Anisotropic damage model}

2.2.1. Damage variable choice. Rock-like materials are frequently weakened by the emergence of micro-cracks and micro-voids, which induce anisotropy for the mechanical behaviours of materials, especially under compression. The isotropic damage model can illustrate the propagation of the micro-defects and softening behaviour of this kind of material (see the third part); however, it cannot account for the anisotropic behaviours of the material. Different anisotropic damage models have been proposed by scholars [43-46]. In this paper, a

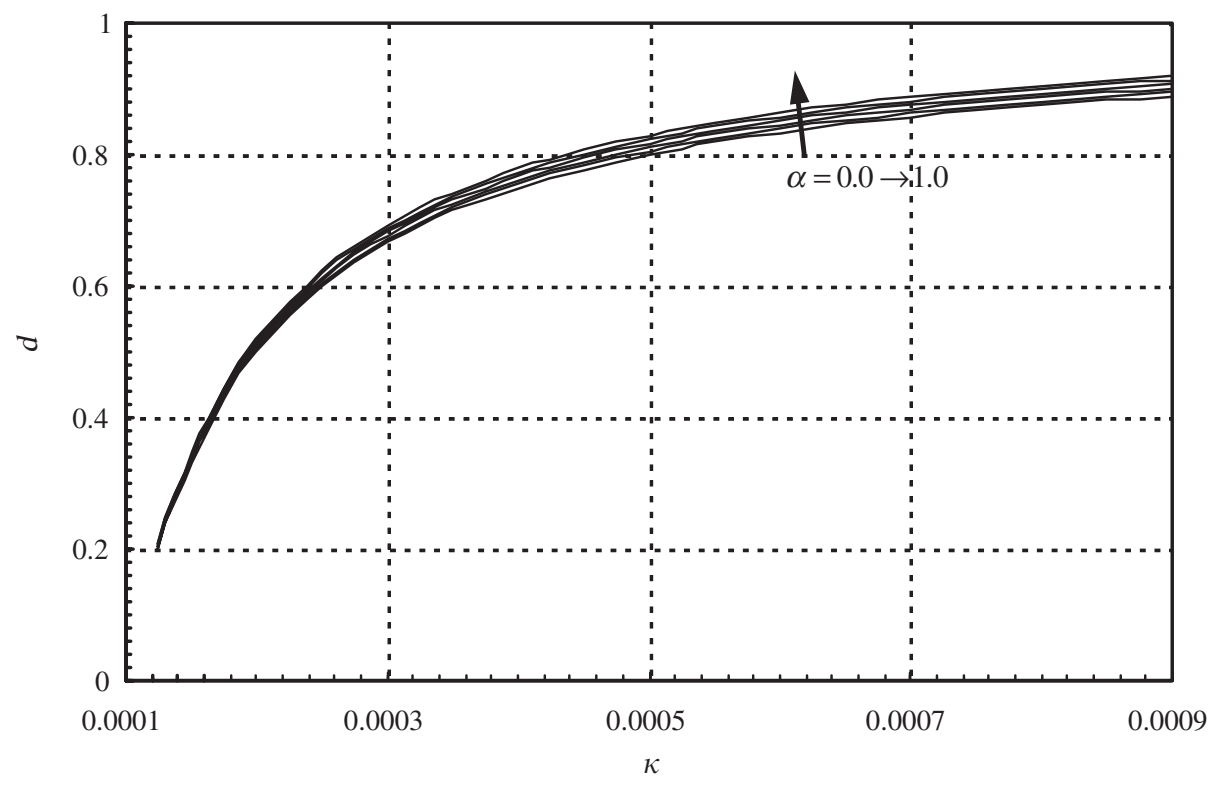

Figure 2. Relation curves for $d$ vs $\kappa$ and $\alpha$ (on condition that $\kappa_{0}=0.001, \zeta=400$ ). 


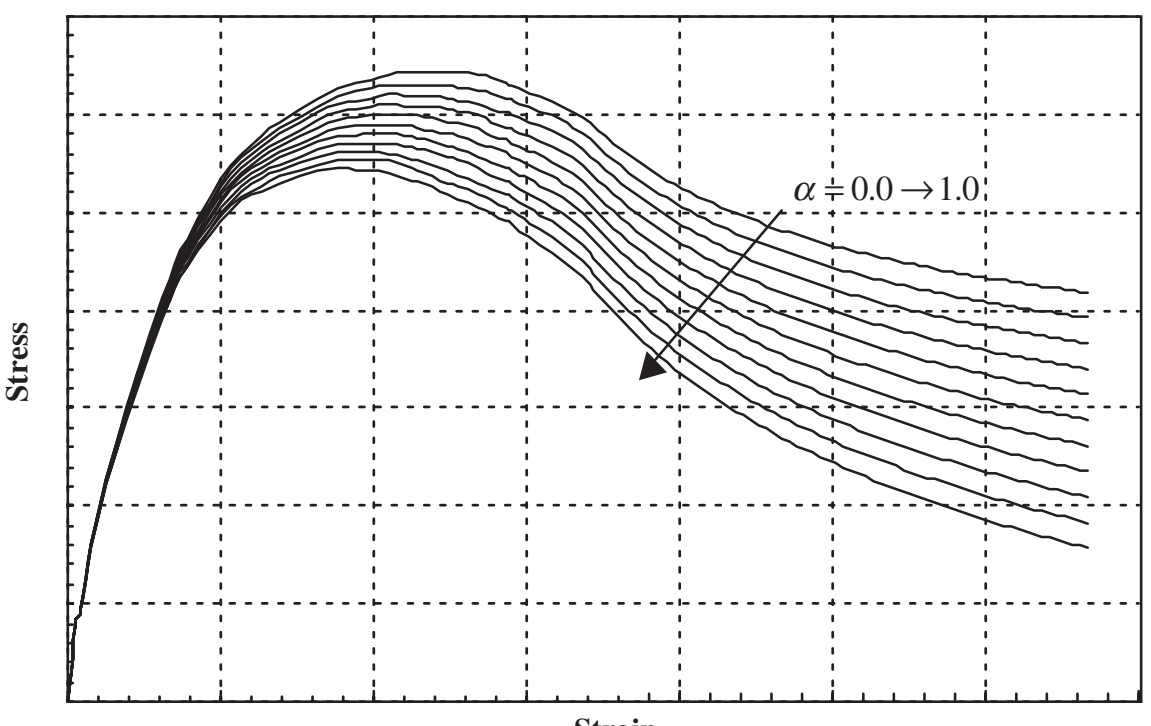

Strain

Figure 3. The effect of $\alpha$ on the curve of uniaxial stress and strain.

second-order damage tensor proposed by Oda [47] and Cowin [48] is used to describe the microdefect-induced anisotropy. Suppose a representative element volume (REV) with a volume of $V^{0}$ involves micro-cracks of total number $n$, the damage behaviour is delineated by the following second-order fabric tensor:

$$
\boldsymbol{\Omega}=\frac{1}{V^{0}} \sum_{\alpha=1}^{n} r_{\alpha}^{3} \mathbf{h}^{\alpha} \mathbf{h}^{\alpha}
$$

where $r_{\alpha}$ and $\mathbf{h}^{\alpha}$ are the radii and the normal unit vector of $\alpha$ th penny-shape micro-crack, respectively. Although the anisotropic damage model in this paper is based on micro-cracks distributed in the material, it is assumed that the interaction of the micro-cracks is taken into account implicitly within the framework of the self-consistent method.

The following expression is used to obtain the elasticity tensors involving damage for the material:

$$
D_{i j k l}=\lambda \Phi_{i j} \Phi_{k l}+\mu\left(\Phi_{i k} \Phi_{j l}+\Phi_{i l} \Phi_{j k}\right), \quad \Phi_{i j}=C_{1} \delta_{i j}+C_{2} \Omega_{i j}+C_{3} \Omega_{i m} \Omega_{m j}
$$

For the sake of convenience, Swoboda and Yang [49] normalized the damage variables and obtained a simple case for the coefficients: $C_{1}=1, C_{2}=-k, C_{3}=-(1-k)$. In this paper, an even simpler case presented by the authors [50] in the case of $k=1$ is used:

$$
\begin{aligned}
D_{i j k l}= & \lambda \delta_{i j} \delta_{k l}+\mu\left(\delta_{i k} \delta_{j l}+\delta_{i l} \delta_{j k}\right)-\left[\lambda \Omega_{i j} \delta_{k l}+\mu\left(\Omega_{i k} \delta_{j l}+\Omega_{i l} \delta_{j k}\right)\right] \\
& +(\lambda+2 \mu) \Omega_{i j} \Omega_{k l}
\end{aligned}
$$


2.2.2. Constitutive relations and damage evolution law. A form of Helmholtz free energy similar to (2) is given for anisotropic behaviour:

$$
\psi=\frac{1}{2} \boldsymbol{\varepsilon}: \mathbf{D}: \boldsymbol{\varepsilon}+\frac{1}{2} \boldsymbol{\eta} \vdots \mathbf{\Lambda} \vdots \boldsymbol{\eta}+\int_{0}^{\kappa} \phi(\kappa) \mathrm{d} \widehat{\kappa}
$$

Suppose the gradient-related elastic tensors still have the form

$$
\boldsymbol{\Lambda}_{i j k l m n}=l^{2} D_{i j l m} \delta_{k n}
$$

Analogous to the isotropic case, the constitutive relations are obtained through ClausiusDuhem inequation

$$
\sigma_{i j}=\frac{\partial \psi}{\partial \varepsilon_{i j}}=D_{i j k l} \varepsilon_{k l}, \quad \tau_{i j k}=\frac{\partial \psi}{\partial \eta_{i j k}}=l^{2} D_{i j k l} \delta_{m n} \eta_{l m n}
$$

Note that the Cauchy stress and higher-order stress tensor above formulated still satisfy the equilibrium equation (4).

The conjugate force of damage can be defined similarly as

$$
\mathbf{Y}=-\frac{\partial \psi}{\partial \boldsymbol{\Omega}}=\frac{1}{2} \boldsymbol{\varepsilon}: \frac{\partial \mathbf{D}(\boldsymbol{\Omega})}{\partial \boldsymbol{\Omega}}: \boldsymbol{\varepsilon}+\frac{1}{2} \boldsymbol{\eta}: \frac{\partial \boldsymbol{\Lambda}(\boldsymbol{\Omega})}{\partial \mathbf{\Omega}} \vdots \boldsymbol{\eta}
$$

From (17) and (19), one can obtain

$$
\begin{gathered}
\frac{\partial D_{i j k l}}{\partial \Omega_{m n}}=-\left(D_{r j k l}^{\mathrm{e}} \delta_{i m} \delta_{n r}+D_{i j k r}^{\mathrm{e}} \delta_{r m} \delta_{n l}\right)+D_{r j k s}^{\mathrm{e}} \Omega_{i r} \delta_{s m} \delta_{n l}+D_{r j k s}^{\mathrm{e}} \Omega_{s l} \delta_{i m} \delta_{n r} \\
\frac{\partial \Lambda_{i j k l a b}}{\partial \Omega_{m n}}=l^{2} \frac{\partial D_{i j l a}}{\partial \Omega_{m n}} \delta_{k b}
\end{gathered}
$$

where $D_{i j k l}^{\mathrm{e}}=\lambda \delta_{i j} \delta_{k l}+\mu\left(\delta_{i k} \delta_{j l}+\delta_{i l} \delta_{j k}\right)$ is the initial elasticity tensor.

A conjugate-force-based damage evolution law is defined as

$$
\dot{\mathbf{\Omega}}=\mathbf{J}: \mathbf{Y}
$$

where $\dot{\boldsymbol{\Omega}}$ is the evolution rate of damage; $\mathbf{J}=\mathbf{J}(\boldsymbol{\Omega})$ is the damage characteristic tensor, a tensor of rank four for a second-order damage tensor. It is defined as

$$
\begin{aligned}
\mathbf{J}(\boldsymbol{\Omega}) & =\sum_{v=1}^{3} \omega_{v}^{2}\left(4 \mathbf{T}_{v}+\frac{9}{4} \mathbf{N}_{v}\right), \quad N_{i j k l}^{v}=m_{i}^{v} m_{j}^{v} m_{k}^{v} m_{l}^{v} \\
T_{i j k l}^{v} & =\frac{1}{2}\left(m_{i}^{v} m_{k}^{v} \delta_{j l}+m_{i}^{v} m_{l}^{v} \delta_{j k}+m_{j}^{v} m_{k}^{v} \delta_{i l}+m_{j}^{v} m_{l}^{v} \delta_{i k}\right)-m_{i}^{v} m_{j}^{v} m_{k}^{v} m_{l}^{v}
\end{aligned}
$$

Here, $\mathbf{m}^{v}$ and $\omega_{v}(v=1,2,3)$ are the principal vector and the principal value of damage tensor $\boldsymbol{\Omega}$, respectively.

It should be noted that, such a conjugate-force-based damage evolution law presented in (26) furnishes a much more general and compact form than does a stress/strain-based one. It possesses the same form in both stress and strain spaces and internal variables. 


\section{NUMERICAL SIMULATION OF DAMAGE LOCALIZATION BY FEM}

\subsection{Element continuity for FEM implementation}

To account for the strain gradient in implementation of FEM, element with $C_{1}$ continuity should be adopted instead of $C_{0}$ continuity element. The $C_{1}$ continuity element was extensively discussed in the geometrically non-linear section by Zienkiewicz [51]. Xia and Hutchinson [52] proposed a triangle element with $C_{1}$ continuity and extended it to elements with more nodes. Wei and Hutchinson [53] studied the effects of strain-gradient plasticity of steady crack tip using this element. Zervos et al. [54] also developed a kind of triangular element of $C_{1}$ continuity with 36 DOF to include the gradient of both elasticity and plasticity, with two different intrinsic length scales for the two periods of constitutive law, respectively. However, they include so many gradient items (both the first order and the second order of strain) that the interpolation and computation of FEM are intensely complicated. Comparably, this paper adopts the method proposed by Xia and Hutchinson [52] to only account for the first gradient of strain, within the framework of brittle damage mechanics for rock-like materials.

Generally, keeping the continuity of first-order gradient of displacement will preserve the $C_{1}$ continuity of an element. Thus, four and six additional DOF are needed for each node for twodimensional and three-dimensional problems, respectively. Figure 4 shows a plain-strain element with 18 DOF, namely, N3U18, developed by Xia and Hutchinson [52]. It is briefly introduced here and it was used in the FE analysis.

The nodal displacement vector for the three nodes of the element is defined as

$$
\mathbf{a}=\left(\begin{array}{lll}
\mathbf{a}_{1} & \mathbf{a}_{2} & \mathbf{a}_{3}
\end{array}\right)^{\prime}, \quad \mathbf{a}_{i}=\left(\begin{array}{llllll}
u & u_{, x} & u_{, y} & v & v_{, x} & v_{, y}
\end{array}\right)_{i}^{t}
$$

The general displacement is supposed to be

$$
\begin{aligned}
u= & \sum_{i=1}^{3} \alpha_{i} L_{1}+\alpha_{4} L_{1} L_{2}+\alpha_{5} L_{2} L_{3}+\alpha_{6} L_{1} L_{3} \\
& +\alpha_{7}\left\{L_{1}^{2} L_{2}+\frac{1}{2} L_{1} L_{2} L_{3}\left[3\left(1-\mu_{3}\right) L_{1}-\left(1+3 \mu_{3}\right)\left(L_{2}-L_{3}\right)\right]\right\}
\end{aligned}
$$

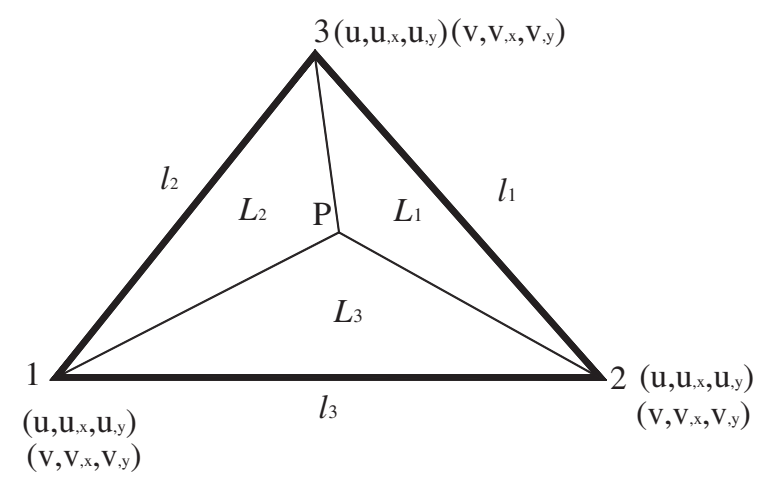

Figure 4. N3U18 triangular element with $C_{1}$ continuum for gradient strain. 


$$
\begin{aligned}
& +\alpha_{8}\left\{L_{2}^{2} L_{3}+\frac{1}{2} L_{1} L_{2} L_{3}\left[3\left(1-\mu_{1}\right) L_{2}-\left(1+3 \mu_{1}\right)\left(L_{3}-L_{1}\right)\right]\right\} \\
& +\alpha_{7}\left\{L_{1}^{2} L_{2}+\frac{1}{2} L_{1} L_{2} L_{3}\left[3\left(1-\mu_{2}\right) L_{3}-\left(1+3 \mu_{2}\right)\left(L_{1}-L_{2}\right)\right]\right\}
\end{aligned}
$$

where $L_{i}$ is the $i$ th area co-ordinate $(i=1,2,3), \alpha_{i}(j=1, \ldots, 9)$ is the coefficient to be determined. $\mu_{i}=\left(l_{k}^{2}-l_{j}^{2}\right) / l_{i}^{2}(i, j, k)$, where $(i, j, k)$ reiterates according to the circle permutations as $(1 \rightarrow 2 \rightarrow 3 \rightarrow 1)$.

The displacement field is expressed in terms of shape function as

$$
u=\sum_{i=1}^{3} N_{i} \mathbf{a}_{i}, \quad N_{i}=\left(\begin{array}{lll}
N_{i}^{1} & N_{i}^{2} & N_{i}^{3}
\end{array}\right)=\left\{\begin{array}{c}
3 L_{i}^{2}-2 L_{i}^{3}-2 L_{i} L_{j} L_{k} \\
-c_{j}\left(L_{i}^{2} L_{k}+L_{i} L_{j} L_{k}\right)+c_{k} L_{i}^{2} L_{j} \\
b_{j}\left(L_{i}^{2} L_{k}+L_{i} L_{j} L_{k}\right)-b_{k} L_{i}^{2} L_{j}
\end{array}\right\}^{t}
$$

where $b_{i}=y_{j}-y_{k}, c_{i}=y_{k}-y_{j}$.

Then the general element strain including the effect of strain gradient appears as the vector form given below:

$$
\begin{aligned}
E=\left(\begin{array}{cccccc}
\varepsilon_{x x} & \varepsilon_{y y} & \gamma_{x y} & \eta_{x x 3} & \eta_{x y 3} & \eta_{y y 3}
\end{array}\right)^{\mathrm{T}}=\mathbf{B a}= \\
\sum_{i=1}^{3}\left\{\begin{array}{cccccc}
N_{i, x}^{1} & N_{i, x}^{2} & N_{i, x}^{3} & 0 & 0 & 0 \\
0 & 0 & 0 & N_{i, y}^{1} & N_{i, y}^{2} & N_{i, y}^{3} \\
N_{i, y}^{1} & N_{i, y}^{2} & N_{i, y}^{3} & N_{i, x}^{1} & N_{i, x}^{2} & N_{i, x}^{3} \\
N_{i, x x}^{1} & N_{i, x x}^{2} & N_{i, x x}^{3} & 0 & 0 & 0 \\
N_{i, y y}^{1} & N_{i, y y}^{2} & N_{i, y y}^{3} & 0 & 0 & 0 \\
N_{i, x y}^{1} & N_{i, x y}^{2} & N_{i, x y}^{3} & 0 & 0 & 0 \\
0 & 0 & 0 & N_{i, x x}^{1} & N_{i, x x}^{2} & N_{i, x x}^{3} \\
0 & 0 & 0 & N_{i, y y}^{1} & N_{i, y y}^{2} & N_{i, y y}^{3} \\
0 & 0 & 0 & N_{i, x y}^{1} & N_{i, x y}^{2} & N_{i, x y}^{3}
\end{array}\right\} \times\left\{\begin{array}{c}
u_{i} \\
(u, x)_{i} \\
(u, y)_{i} \\
v_{i} \\
(v, x)_{i} \\
(v, y)_{i}
\end{array}\right\}
\end{aligned}
$$

where $\mathbf{B}$ is the strain-displacement matrix.

The extension of $C_{1}$ continuity finite element to three-dimensional case is straightforward. Based on the idea of plane triangular elements, 3D tetrahedron element, 3D cubic element and triangular prism element of $C_{1}$ continuity have been constructed by the authors. Further development of various variable-nodal 3D elements, such as 8 nodal and 9-20 nodal hexahedron elements, are still under study and will soon be applied to the 3D FEM simulation of gradient model. 


\subsection{Finite difference method (FDM) coupled with FEM for regular rectangle element}

When elements in FEM computation are all regular rectangles, a simple coupled method between FEM and FDM can be achieved. The advantages of this method have the following points: (1) simple for computation; (2) little effort is required to modify the normal FEM code; (3) high computation efficiency; (4) no larger loss of precision compared with FEM for general simple cases. These can be seen from a comparison in Section 3.3. The main idea is a combination of nodal gradient obtained by FDM into the strain of FEM computation. Here an illustration is given.

As sketched in Figure 5, rectangular plane-strain elements are considered. In this case, only $\eta_{113}, \eta_{223}, \eta_{123}$ and $\eta_{213}$ are non-zero among the components of $\eta_{i j k}$ for each node. From the theory of finite difference method, for a variable $u$, when the difference pace $\Delta x$ is infinitesimal, the first derivative can be approximated as

$$
\frac{\mathrm{d} u}{\mathrm{~d} x} \doteq \frac{u(x+\Delta x)-u(x)}{\Delta x}
$$

According to the definition of strain gradient of Equation (2), the strain gradient of the node $(i, j)$ in Figure 3 can be determined similarly

$$
\begin{gathered}
\eta_{113}^{i, j}=\frac{u_{i+1, j}-2 u_{i, j}+u_{i-1, j}}{(\Delta x)^{2}}, \quad \eta_{223}^{i, j}=\frac{v_{i, j+1}-2 v_{i, j}+v_{i, j-1}}{(\Delta y)^{2}} \\
\eta_{123}^{i, j}=\frac{u_{i+1, j+1}-u_{i-1, j}-u_{i+1, j-1}+u_{i-1, j-1}}{4 \Delta x \Delta y}, \\
\eta_{213}^{i, j}=\frac{v_{i+1, j+1}-v_{i+1, j-1}-v_{i-1, j+1}+v_{i-1, j-1}}{4 \Delta x \Delta y}
\end{gathered}
$$

Since strain gradient tensor is symmetric, $\eta_{123}=\eta_{213}$. The above-approximated difference may not necessarily preserve this symmetry. Therefore, their average values are going to be

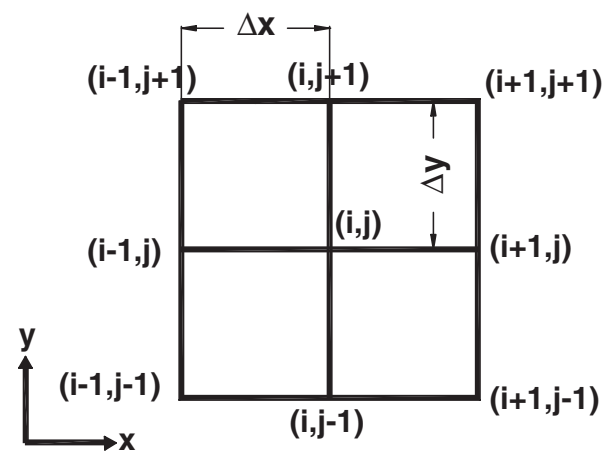

Figure 5. A sketch of the FDM determination of the strain gradient for each node. 
adopted as their final values:

$$
\begin{aligned}
\eta_{123}^{i, j} & =\eta_{213}^{i, j} \\
& =\frac{u_{i+1, j+1}-u_{i-1, j}-u_{i+1, j-1}+u_{i-1, j-1}+v_{i+1, j+1}-v_{i+1, j-1}-v_{i-1, j+1}+v_{i-1, j-1}}{8 \Delta x \Delta y}
\end{aligned}
$$

Note that for each mid-boundary node, the gradient is obtained by difference on the two neighbouring collinear nodes. For each corner node, the gradients are obtained by averaging the gradient of two nearest neighbouring nodes.

Then the elemental strain gradient can be interpolated by the same shape function of FEM for each element

$$
\boldsymbol{\eta}=\left\{\begin{array}{l}
\eta_{113} \\
\eta_{223} \\
\eta_{123}
\end{array}\right\}=\mathbf{N}\left\{\begin{array}{l}
\eta_{113}^{\mathrm{e}} \\
\eta_{223}^{\mathrm{e}} \\
\eta_{123}^{\mathrm{e}}
\end{array}\right\}=\mathbf{N} \boldsymbol{\eta}^{\mathrm{e}}
$$

However, disadvantages of the above method are obvious: (1) only applicable for regular rectangle element; (2) large precision loss for complicate problems.

\subsection{FEM simulation of localization}

3.3.1. Uniaxial tension test: for the same problem presented in Figure 1. The isotropic straingradient-enhanced damage model presented in this paper was used to simulate the same problem in Figure 1. A rectangle $C_{1}$ continuity element N4U24, similar to N3U18 element presented by Xia and Hutchinson [49], is developed. To compare with the traditional results, the combining effects of strain with strain gradient should be considered instead of strain alone. The following form of equivalent strain proposed by Fleck and Hutchinson [25] is adopted:

$$
E=\sqrt{\frac{2}{3} \varepsilon_{i j} \varepsilon_{i j}+l^{2} \eta_{i j k} \eta_{i j k}}
$$

Figure 6 shows the simulated results by N4U24 element incorporated into the isotropic gradient-damage model. Figure 7 shows the results of damage distribution for both models. The chosen parameters of the material for simulations of Figures 1 and 6 are all the same: (a) size: $33 \mathrm{~cm}$ in length and $3 \mathrm{~cm}$ in width; (b) Lamé constants: $\mu=9.0 \times 10^{9} \mathrm{Mpa}, \lambda=6.0 \times 10^{9} \mathrm{Mpa}$; (c) value of $\alpha$ in expression (13) is assumed to be 0.94 , and $\zeta, 400$. The values of damage threshold, $\kappa_{0}$, for all the elements are assumed as 0.0001 , except for those of the middle row at the value of 0.00009 , which generates an agitation for localization.

Obvious differences can be found between Figures 1 and 6, Figure 7(a) and 7(b). Using the isotropic model presented in this paper, the distribution of equivalent strain and damage including strain gradient is nearly independent of the FEM mesh, which shows the great advantage of incorporating strain gradient and intrinsic material length.

3.3.2. Comparison between method using $C_{1}$ continuity element and FDM-FEM mixed method. Computations by using N4U24 $C_{1}$ continuity element and FDM-FEM coupling method are implemented separately. The same mesh of (a) in Figure 4 is used. A comparison of equivalent strain along the $Y$ direction for the two methods is shown in Figure 8. As can be seen, 


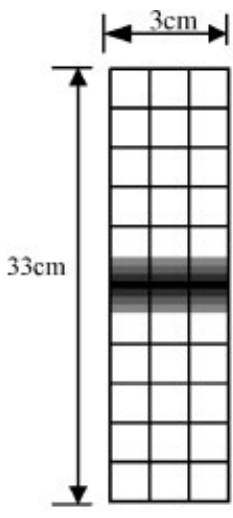

(a) : $3 \times 11$

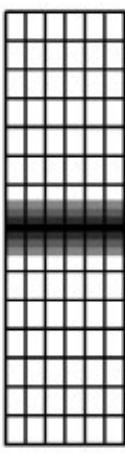

(b) : $6 \times 15$

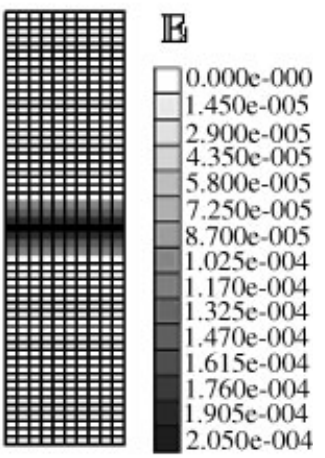

(c) : $10 \times 55$

Figure 6. Uniaxial tension sample: the obtained distribution of equivalent global strain independent of the mesh division with the application of isotropic strain-gradient-enhanced damage model.

no significant loss of precision is caused by coupled FEM-FDM method, when its results were compared with that of FEM computation with $C_{1}$ continuity element. For this simple problem, the time consumptions of computation by coupled method and $C_{1}$ continuity element method are 87 and $124 \mathrm{~s}$, respectively. With the former method, the efficiency of computation is promoted by approximately $43 \%$. However, great deviation may occur when dealing with complicated problems, and in those cases, the difference-obtained gradient will no longer be sufficiently accurate, and special attention should be paid to that issue.

3.3.3. Compression test in plane strain by the anisotropic model. The damage localization of compression test in plain strain by the anisotropic gradient-damage model is demonstrated in Figure 9, in which three different meshes are used to test the mesh-sensitive characteristic. The samples have identical size of $16 \mathrm{~cm} \times 8 \mathrm{~cm}$ (height $\times$ width) and the discretized meshes are $8 \times 9,16 \times 16,24 \times 24$, respectively. Two sets of micro-cracks are considered with the averaged trace length and orientation, $\tilde{r}^{(1)}=\tilde{r}^{(2)}=0.08 \mathrm{~mm}, \theta^{(1)}=45^{\circ}, \theta^{(2)}=-45^{\circ}$. Total cracks for each set are numbered 20. However, microcracks are not simulated directly in the mesh. They are used only to obtain a general damage tensor for the computation. A disturbing imperfection, with a little lower principal value of the damage tensor, is set for the centre elements of each sample to trigger localization. Figure 10 illustrates the comparison of load-displacement curve for the three samples. As can be seen from Figures 9 and 10, obvious localized damage shear bands are found under compression. The simulated results are nearly free of mesh dependence and the localized shear bands are almost of the same size and orientation.

\section{ENGINEERING APPLICATION}

The above-proposed models were also applied to the failure analysis of dam engineering by nonlinear FEM. The engineering case chosen in this paper is Ertan arch dam. 

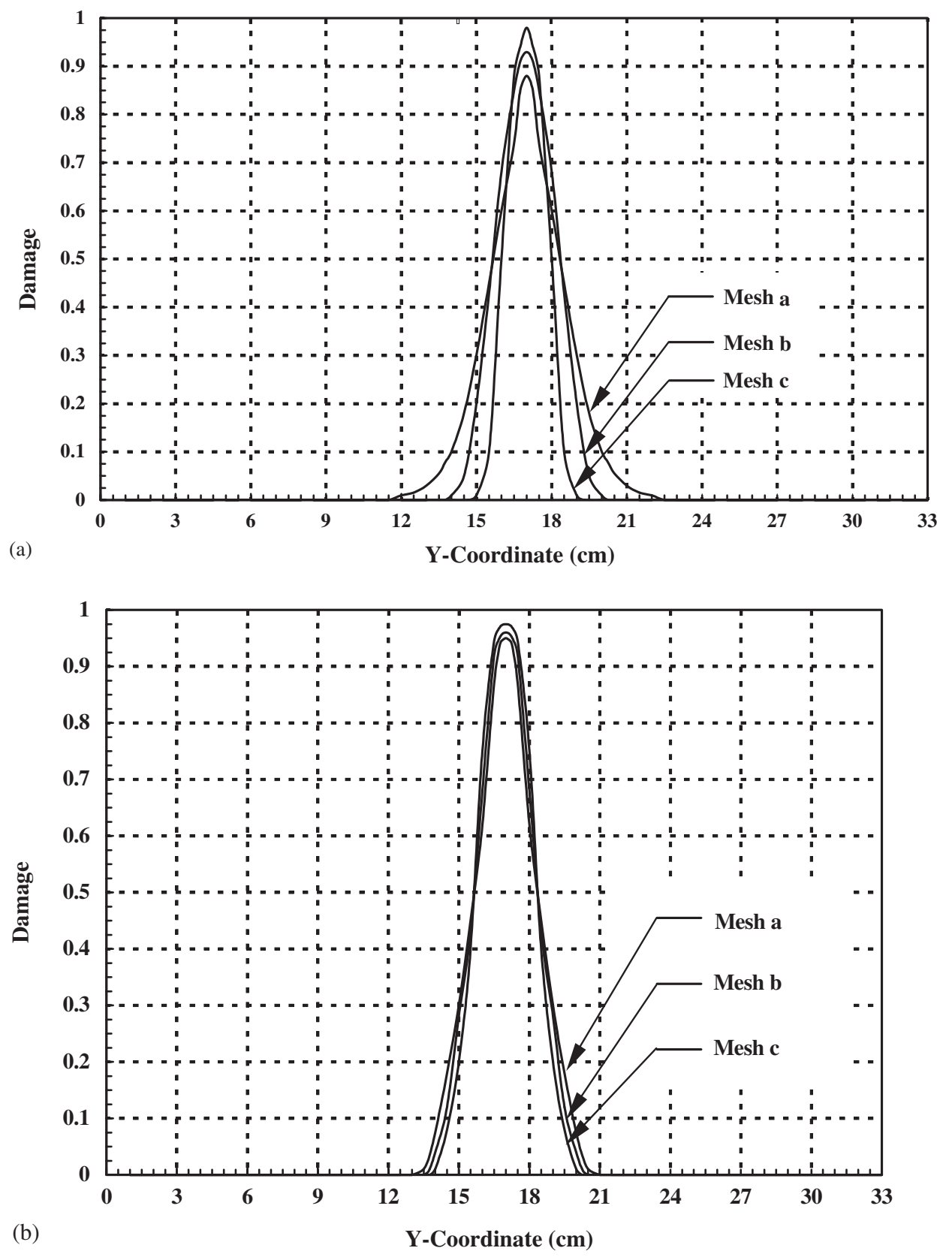

Figure 7. Damage distribution along the $Y$ direction for samples in Figures 1 and 6: (a) traditional damage model; (b) isotropic strain-gradient-enhanced damage model. 


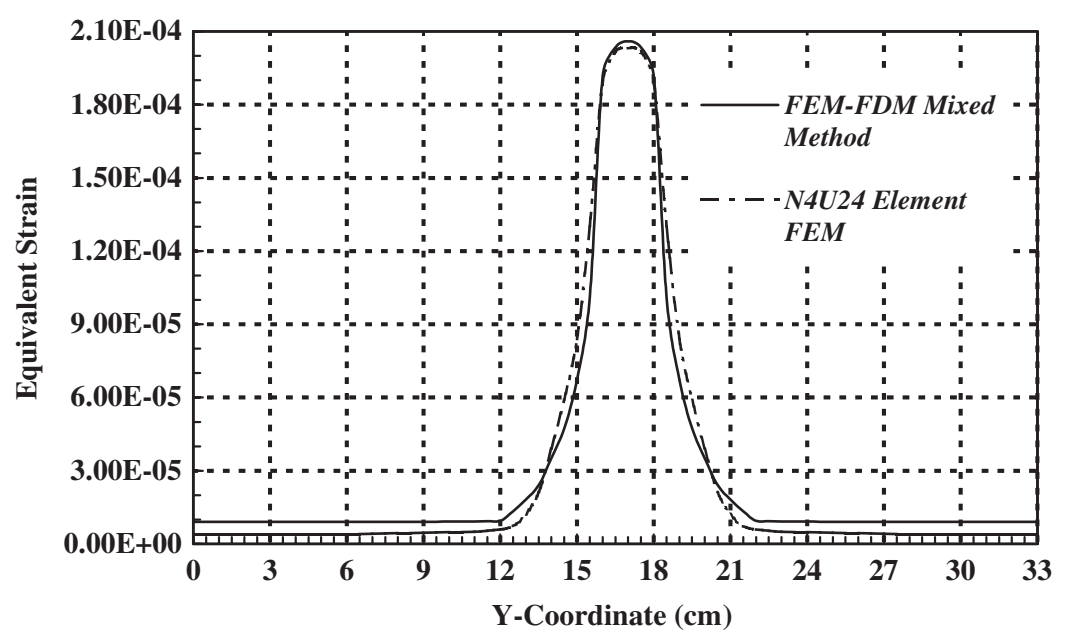

Figure 8. Comparison of nodal effective strain between FEM-FDM mixed method and N4U24 continuum element method (Mesh: $3 \times 11$ ).

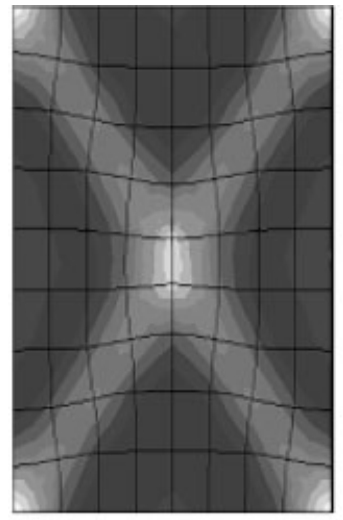

(a)

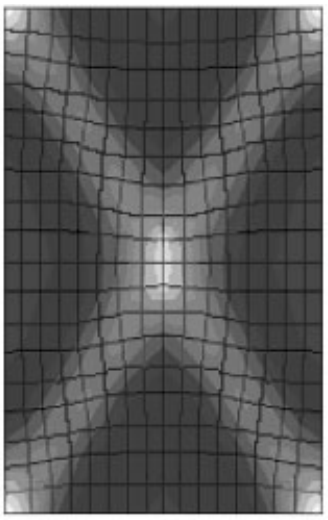

(b)

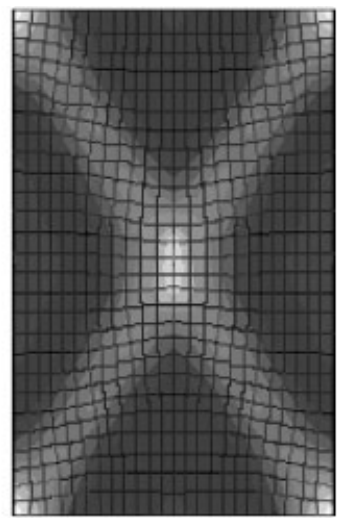

(c)

Figure 9. Damage distribution of compression test (with imperfection in the centre): Mesh (a): $8 \times 9$; Mesh (b): $16 \times 16$; Mesh (c): $24 \times 24$.

The Ertan arch dam is located in the downstream reach of the Yalong River in southwestern China. It is the highest arch dam in China at present. The rock mass at the dam site is generally composed of igneous crystalline rocks. The reservoir was impounded and flood was discharged in June 1998. Characterized data of the project are listed in Table I.

Geophysical model tests, a means to verify the limit capacity under static structural loads and ultimate failure modes for arch dams, have been conducted during the construction of the dam. The models were reproduced to simulate the dam itself, with the dam geological foundations. The model scale is 1:100. Three-dimensional FEM implementation of the proposed anisotropic 


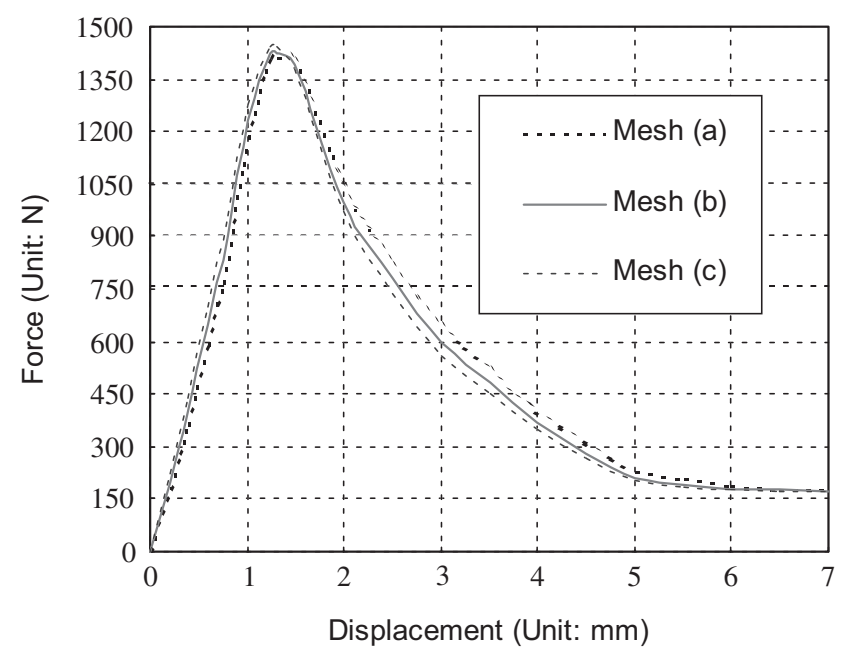

Figure 10. Comparison of the load-displacement curves of the compression tests in Figure 9.

Table I. Characterized data of the Ertan project.

\begin{tabular}{|c|c|c|c|}
\hline Ertan project & & Dam body & \\
\hline Location & Yalong river, China & Dam type & $\begin{array}{l}\text { Double curvature } \\
\text { arch dam }\end{array}$ \\
\hline Main function & Power generation & Max. height & $240 \mathrm{~m}$ \\
\hline $\begin{array}{l}\text { Total installed } \\
\text { capacity }\end{array}$ & $3300 \mathrm{MW}$ & Crest length & $774.69 \mathrm{~m}$ \\
\hline $\begin{array}{l}\text { Annual power } \\
\text { output }\end{array}$ & $17000 \mathrm{GM} \mathrm{h}$ & Thickness at the base of crown & $58.51 \mathrm{~m}$ \\
\hline $\begin{array}{l}\text { Project } \\
\text { configuration }\end{array}$ & $\begin{array}{l}\text { Arch dam, water releasing } \\
\text { structure, diversion channel, } \\
\text { switch station, log-passing } \\
\text { system and secondary dam }\end{array}$ & $\begin{array}{l}\text { Thickness at the top of crown } \\
\text { Max central angle of arch } \\
\text { Max. overhang ratio of upstream } \\
\text { Curvature radii range }\end{array}$ & $\begin{array}{l}11 \mathrm{~m} \\
91.5^{\circ} \\
0.18 \\
349.19-981.15 \mathrm{~m}\end{array}$ \\
\hline
\end{tabular}

model in this paper was conducted to account for the behaviours of complex geological and fracture conditions of the bed rock mass. Altogether 2523 elements are simulated, and among them, 523 elements are dam body elements. The 3D FE mesh can be seen from Figure 11(a). Altogether 31 materials with different parameters are used to account for the complicate geological conditions, including several large faults and millions of micro-fracture in the bed rock mass.

From geophysical model tests, the dam body and dam foundation collapses under the pressure of 7 times normal water load, which coincides rather well with that from the FEM computation. Moreover, from the final failure modes of geophysical model tests in Figure 11(c), there is an intensified, elliptical-shape-like collapse zone in the middle of the dam body (grey coloured zone), which is similar to that of the final damage distribution of FEM computation in Figure 11(b). Therefore, the results from geophysical model tests and FEM numerical 
simulations were mutually verified, and both may be served as useful tools for engineering failure evaluation and prediction. Thus, it is verified that the models in this paper can make sense both in theory and practical applications.

(a)
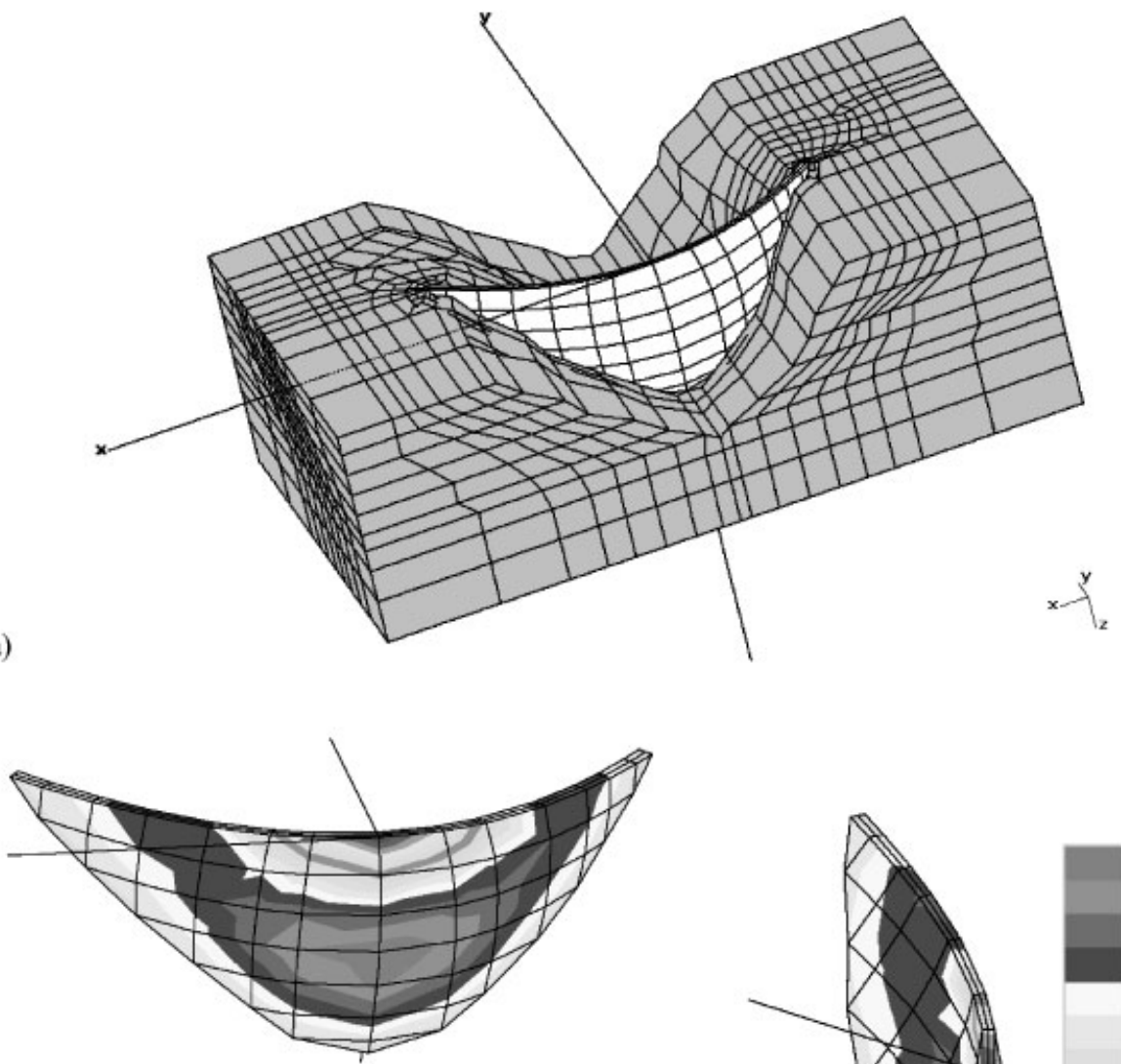

(b-1) Upstream View
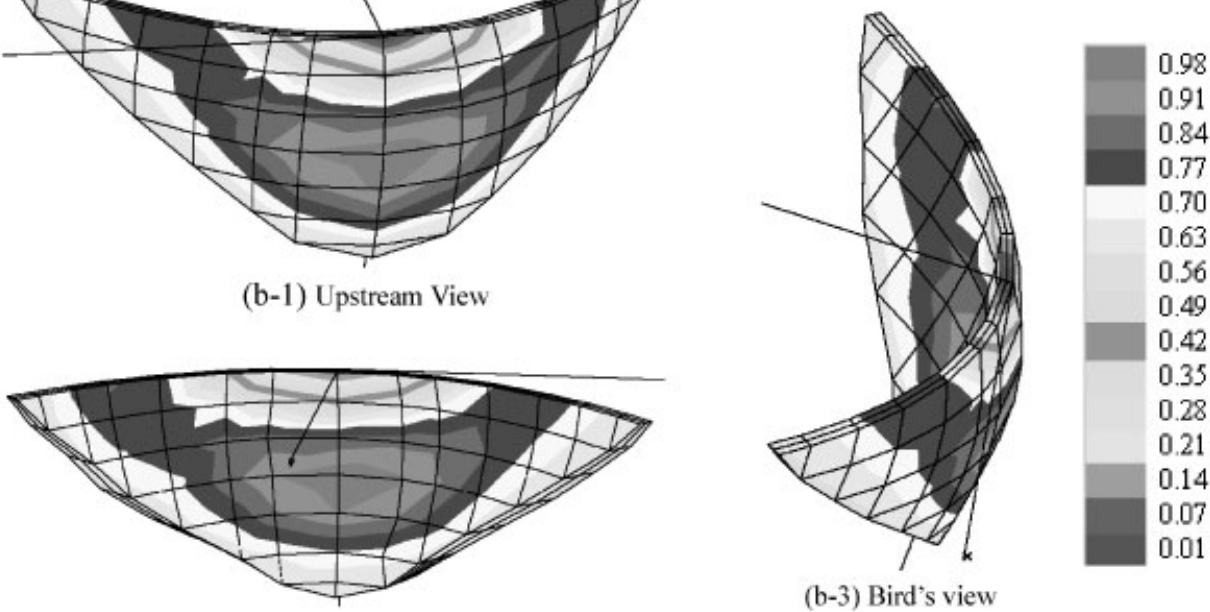

(b)

(b-2)Downstream View

(b-3) Bird's view

Figure 11. Comparison between FEM analysis and geophysical model test for the failure behaviour of Ertan arch dam: (a) FE Mesh of Ertan arch dam and the bed rock mass; (b) damage distribution of the dam body obtained by FEM analysis under 7 times overloading; (c) Rupture behaviours by geophysical model test (conducted in 1994). 


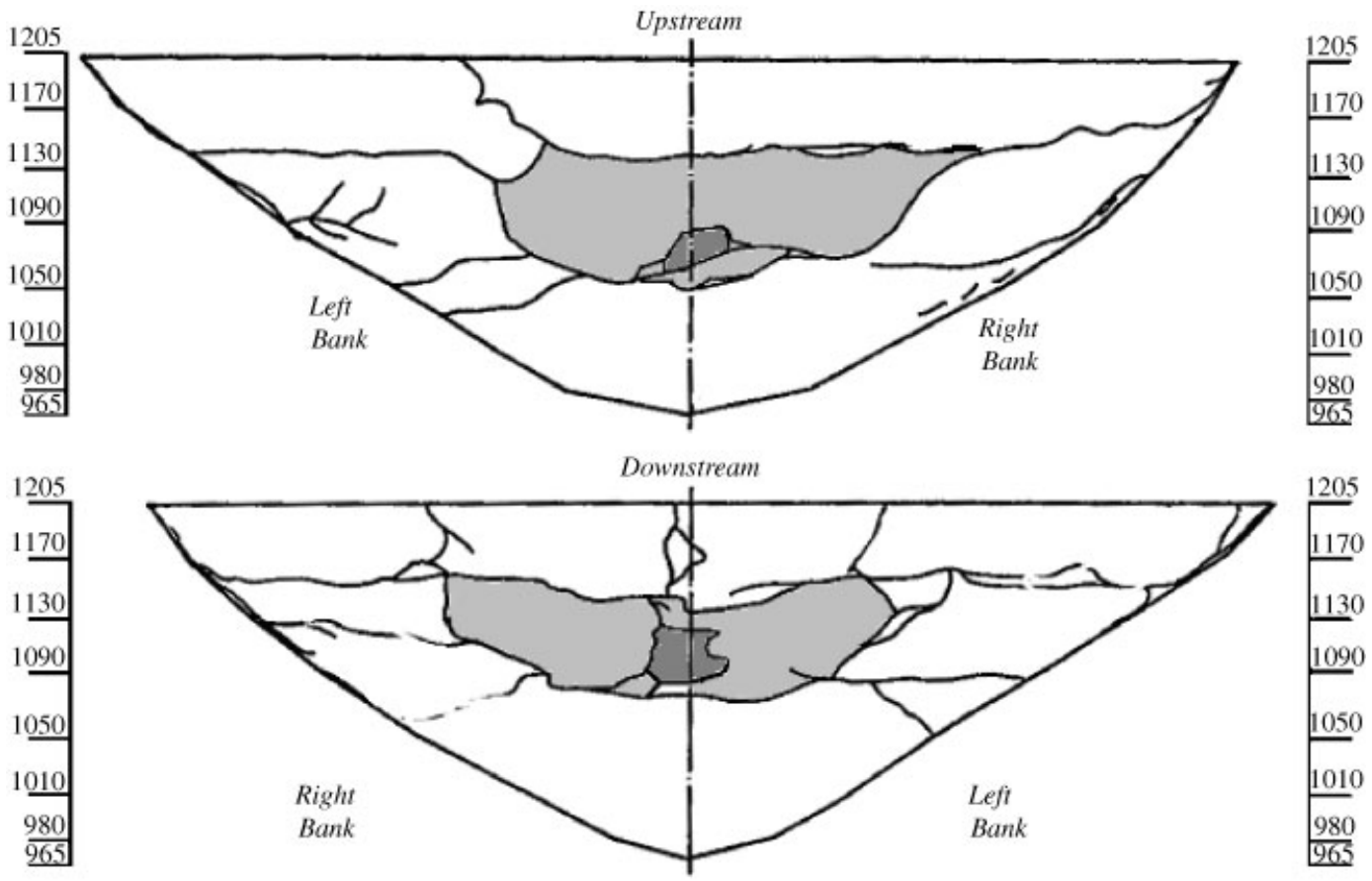

(c)

Figure 11. (continued)

\section{CONCLUSIONS}

The strain-gradient-enhanced damage model presented in this paper can properly characterize and describe the phenomena of strain localization in rock-like materials. It can solve the problem of mesh dependence in FEM simulation and avoid the loss of well posedness of the governing equation in traditional damage models. To avoid a great modification of the procedure of FEM implementation, the results of strain gradient by finite difference methods are combined into the FEM procedure. However, a disadvantage of this approach is that the requirement for mesh discretization is overly rigorous, thus its application is limited. The anisotropic model can well illustrate the anisotropic characteristic of localized damage for rock-like materials, which frequently exhibit great anisotropic behaviours due to the existence of large quantities of micro-cracks and micro-voids. Application of the anisotropic model to the 3D-non-linear FEM analysis of Ertan arch dam was conducted and the results of numerical simulation of the failure behaviours coincide well with those obtained by geophysical model test of the dam. However, the uncertainty of intrinsic length scale associated with strain gradient and damage needs further experimental and theoretical studies. 


\section{ACKNOWLEDGEMENTS}

This research was financially sponsored by the National Science Foundation of China (NSFC) through project No. 59939190 and Schlumberger BGC. The authors also wish to send acknowledgements to Prof. Yang Ruoqiong (Tsinghua University, People's Republic of China) for her generous help and technical assistance. The second author wants to thank Dr Wu Rongzong (UC Berkeley, USA) for the helpful discussions with him.

\section{REFERENCES}

1. Rudnicki JW, Rice JR. Conditions for the localization of deformation in pressure-sensitive dilatant materials. Journal of the Mechanics and Physics of Solids 1975; 23:371-395.

2. Ottosen NE, Runesson K. Properties of discontinuous bifurcation solutions in elasto-plasticity. International Journal of Solids and Structures 1991; 27(4):401-421.

3. Benallal A. On localization phenomena in thermo-elasto-plasticity. Archives of Mechanics 1992; 44(1):15-29.

4. Comi C. Computational modelling of gradient-enhanced damage in quasi-brittle materials. Mechanics of CohesiveFrictional Materials 1999; 4(1):17-36.

5. Fremond M, Nedjar B. Damage, gradient of damage and principle of virtual power. International Journal of Solids and Structures 1996; 33(8):1083-1103.

6. Muhlhaus HB, Vardoulakis I. The thickness of shear bands in granular materials. Geotechnique 1987; 37:217-283.

7. De Borst R, Muhlhaus HB. Gradient dependent plasticity: formulation and algorithmic aspects. Journal of Numerical Methods in Engineering 1992; 35:521-539.

8. Bažant ZP, Oh BH. Crack band theory for fracture of concrete. RILEM Materials and Structures 1983; 16:155-177.

9. Bažant ZP, Belytschko B, Chang TP. Continuum theory for strain softening. Journal of Engineering Mechanics 1984; 110(12):1666-1693.

10. Bažant Z, Ozbolt J. Nonlocal microplane model for fracture damage and size effect in structures. Journal of Engineering Mechanics 1990; 116:2485-3505.

11. Bažant ZP, Jaime Planas. Fracture Size Effect in Concrete and Other Quasi-brittle Materials. CRC Press LLC: Florida, U.S.A., 1998.

12. Peerlings RHJ, de Borst R, Brekelmans WAM, Vree JHP. Gradient enhanced damage for quasi-brittle materials. International Journal for Numerical Methods in Engineering 1996a; 39:3391-3404.

13. Peerlings RHJ, de Borst R, Brekelmans WAM, Vree JHP. Some observations on localization in non-local and gradient damage models. European Journal of Mechanics Part A/Solids 1996b; 15:937-953.

14. Peerlings RHJ, de Borst R, Brekelmans WAM, Geers MGD. Gradient enhanced modeling of concrete fracture. Mechanics of Cohesive-Frictional Materials 1998; 3:323-342.

15. Geers MGD, de Borst R, Brekelmans WAM, Peerlings RHJ. Strain-based transient-gradient damage model for failure analyses. Comparative Methods in Applied Mechanics and Engineering 1998a; 160(1-2):133-154.

16. Geers MGD, de Borst R, Brekelmans WAM, Peerlings RHJ. Validation and internal length scale determination for a gradient damage model: application to short glass-fiber-reinforced polypropylene. International Journal of Solids and Structures 1998b; 36:2557-2583.

17. Kuhl Ellen, Ramm Ekkehard. Simulation of strain localization with gradient with gradient enhanced damage models. Computational Materials Sciences 2000; 16:176-185.

18. Tvergaard V, Needleman A. Effects of non-local damage in porous plastic solids. International Journal of Solids and Structures 1995; 32(8/9):1063-1077.

19. Cosserat E, Cosserat F. Theorie des Corps Deformables. A. Hermann\& files: Paris, 1909.

20. Toupin RA. Elastic materials with couple stresses. Archive for Rational Mechanics and Analysis 1962; 11:385-414.

21. Mindlin RD. Micro-structure in linear elasticity. Archive for Rational Mechanics and Analytic 1964; 16:51-78.

22. Mindlin RD. Second gradient of strain and surface tension in linear elasticity. International Journal on Solids and Structures $1965 ; \mathbf{2 8}: 845-857$.

23. Aifantis EC. On the micro-structural origin of certain inelastic models. Transactions of ASME Journal of Engineering Materials and Technics 1984a; 106:326-330.

24. Aifantis EC. Remarks on media with microstructures. International Journal of Engineering Science 1984b; 22: 961-968.

25. Muhlhaus HB, Aifantis EC. A variational principle for gradient plasticity. International Journal Solids and Structures 1991; 28:845-857.

26. Fleck NA, Hutchinson JW. A phenomenological theory for strain gradient effects in plasticity. Journal of Mechanics and Physics of Solids 1993; 41(12):1825-1857.

27. Fleck NA, Hutchinson JW. Strain gradient plasticity. In Advances in Applied Mechanics, Vol. 33, Hutchinson JW, Wu TY (eds). Academic Press: New York, 1997; 295-361. 
28. Fleck NA, Muller GM, Ashby MF, Hutchinson JW. Strain gradient plasticity: theory and experiment. Acta Metallurgica et Materialia 1994; 42:475-487.

29. Gao H, Huang Y, Nix WD. Modeling plasticity at the micrometer scale. Naturwissenschaftlen 1999; 86:507-515.

30. Pan Yishan. Studies on the process of rock burst and failure. Doctoral Thesis, Tsinghua University, China, Beijing, 1999.

31. Xu Songlin, Wu Wen, Li Ting, Wu Yushan, Bai Shiwei. Experimental studies on localization and bifurcation behaviours of a marble under triaxial compression. Chinese Journal of Geotechnical Engineering 2001; 23(3):296-301.

32. Simo J, Ju JW. Strain and stress based continuum damage models: Part I-Formulation, Part II-Computational aspects. International Journal of Solids and Structures 1987; 23:821-869.

33. Shu JY, Fleck NA. Strain gradient crystal plasticity: size-dependent deformation of bicrystals. Journal of the Mechanics and Physics of Solids 1999; 47:297-324.

34. Stolken JS, Evans AG. A microbend test method for measuring the plasticity length scale. Acta Materialia 1998; 46:5109-5115.

35. Nix WD. Mechanical properties of thin films. Metallic Transactions A 1989; 20A:2217-2245.

36. De Guzman MS, Neubauer G, Flinn P, Nix WD. The role of indentation depth on the measured hardness of materials. Materials Research Symposium Proceedings 1993; 308:613-618.

37. Stelmashenko NA, Walls MG, Brown LM, Milman YV. Microindentation on W and Mo orientated single crystals: an STM study. Acta Metallurgica et Materialia 1993; 41:2855-2865.

38. Ma Q, Clarke DR. Size dependent hardness of silver single crystals. Journal of Materials and Researches 1995; 10:853-863.

39. Poole WJ, Ashby MF, Fleck NA. Micro-hardness of annealed and work-hardened copper polycrystals. Scripta Metallurgica et Materialia 1996; 34:559-564.

40. McElhaney KW, Vlassak JJ, Nix WD. Determination of indenter tip geometry and indentation contact area for depth-sensing indentation experiments. Journal of Materials and Researches 1998; 13:1300-1306.

41. Nix WD, Gao HJ. Indentation size effects in crystalline materials: a law for strain gradient plasticity. Journal of the Mechanics and Physics of Solids 1998; 46:411-425.

42. Carmeliet J, de Borst R. Stochastic approaches for damage evolution in standard and non-standard continua. International Journal of Solids and Structures 1995; 32(8-9):1149-1160.

43. Leckie FA, Onat ET. Tensorial nature of damage measuring internal variables. In Proceedings of IUTAM Symposium on Physical Nonlinearities in Structural Analysis. Hult J, Lemaitre JL (eds). Springer: Senlis, France, $1981 ; 140-155$.

44. Carol I, Bazant ZP, Prat PC. Geometric damage tensor on microplane model. ASCE Journal of Engineering Mechanics 1991; 117:2429-2448.

45. Lemaiter J, Desmorat R, Sauzay M. Anisotropic damage law of evolution. European Journal of Mechanics A/Solids 2000; 19:187-208.

46. Shao JF, Rudnicki JW. A microcrack-based continuous damage model for brittle geomaterials. Mechanisms of Materials 2000; 32:607-619.

47. Oda M, Suzuki K, Maeshibu T. Elastic compliance for rock-like materials with random cracks. Solids and Foundations 1984; 24:27-40.

48. Cowin SC. The relationship between the elasticity tensor and the fabric tensor. Mechanisms of Materials 1985; 4:137-147.

49. Swoboda G, Yang Q. An energy-based damage model of geomaterials-I. Formulation and numerical results. International Journal of Solids and Structures 1999; 36:1719-1734.

50. Zhou Weiyuan, Yang Yanyi. A fracture damage model for jointed rock masses and its application to the stability analysis of dam abutments. International Conference on Constitutive Laws for Engineering Materials, Chongqing, China, 1989; 8.

51. Zienkiewicz OC. The Finite Element Method, Chapter 19. McGraw-Hill: UK, 1977; 500-526.

52. Xia ZC, Hutchinson JW. Crack tip fields in strain gradient plasticity. Journal of the Mechanics and Physics of Solids 1996; 44(10):1621-1648.

53. Wei Y, Hutchinson JW. Steady-state crack growth and work of fracture for solids characterized by strain gradient plasticity. Journal of the Mechanics and Physics of Solids 1997; 45(8):1253-1273.

54. Zervos A, Papanastasiou P, Vardoulakis PI. Modeling of localization and scale effect in thick-walled cylinders with gradient elastoplasticity. International Journal of Solids and Structures 2001; 38:5081-5095. 